\title{
A Simple Yet Effective Graph Partition Model for GPU Computing
}

\author{
Eddy Z. Zhang \\ Rutgers University \\ zz124@cs.rutgers.edu
}

\begin{abstract}
Graph edge partition models have recently become an appealing alternative to graph vertex partition models for parallel and distributed computing due to their flexibility in balancing loads and their performance in reducing communication cost $[1,3]$. In this paper, we introduce a simple yet effective graph edge partitioning model for GPU computing. In practice, our model yields high partition quality (better than or the same as the state-of-the-art edge partition approaches, at least for power-law graphs) with low partition overhead. In theory, previous work [1] showed that an approximation factor of $O\left(d_{\max } \sqrt{\log n \log k}\right)$ apply to the graphs with $m=\Omega\left(k^{2}\right)$ edges ( $k$ is the number of partitions). Our model extends this result to all graphs.

We demonstrate how graph edge partition model can be applied to GPU computing. We draw our examples from GPU program for locality enhancement both over time and (processor) space. For the first time, we demonstrate the effectiveness of edge partition for modeling data reuse in a many-core processors, both in theory and in practice.
\end{abstract}

\section{CCS CONCEPTS}

- Mathematics of computing $\rightarrow$ Graph algorithms; • Theory of computation $\longrightarrow$ Parallel computing models; $\bullet$ Computing methodologies $\rightarrow$ Modeling and simulation;

\section{KEYWORDS}

Graph partition model; GPU; data reuse; locality

ACM Reference Format:

Eddy Z. Zhang. 2018. A Simple Yet Effective Graph Partition Model for GPU Computing. In Proceedings of 47th International Conference on Parallel Processing Companion (ICPP'18 Comp). ACM, New York, NY, USA, Article 4, 3 pages. https://doi.org/10.1145/3229710.3229721

\section{MODEL OVERVIEW}

Graph edge partition models have recently become an appealing alternative to graph vertex partition models for parallel and distributed computing due to their flexibility in balancing workloads and their performance in reducing communication cost $[1,3]$. In the edge partition model, a graph is partitioned based on the edges

Permission to make digital or hard copies of all or part of this work for personal or classroom use is granted without fee provided that copies are not made or distributed for profit or commercial advantage and that copies bear this notice and the full citation on the first page. Copyrights for components of this work owned by others than ACM must be honored. Abstracting with credit is permitted. To copy otherwise, or republish, to post on servers or to redistribute to lists, requires prior specific permission and/or a fee. Request permissions from permissions@acm.org.

ICPP'18 Comp, August 13-16, 2018, Eugene, OR, USA

(C) 2018 Association for Computing Machinery.

ACM ISBN 978-1-4503-6523-9/18/08...\$15.00

https://doi.org/10.1145/3229710.3229721 as opposed to vertices in the vertex partition problem. The edge partition model optimizes the number of vertex copies, while the vertex partition model optimizes the number of edge cuts.

We use the computation fluid dynamics (CFD) program to demonstrate use of edge partition model for parallel computing (in Figure 1). In $C F D$, the main computation is to calculate the interaction between fluid elements bounded by certain spatial distances and to use the interaction information to update the status of every fluid element for the next time step.

A fluid element is modeled as a vertex. An interaction is calculated between a pair of fluid elements, modeled as an edge. Figure 1 shows six interaction edges among six fluid elements.

Figure 1(a) shows one possible way to partition the computation (edges) to two multi-core processors, in which case, edges $e_{1}, e_{2}, e_{3}$ are mapped to one multi-core processor and $e_{4}, e_{5}, e_{6}$ are mapped to the other multi-core processor, requiring three fluid elements to be copied into both multi-core processors (marked as solid circles). However, with an optimized partition as illustrated in Figure 1(b), with $e_{1}, e_{2}, e_{4}$ mapped to one multi-core processor and $e_{3}, e_{5}, e_{6}$ mapped to the other multi-core processor, only one fluid element needs to be copied into both multi-core processors. In total, Figure 1 (b) reduces the number of vertex copies by $66 \%$, corresponding to a significant memory communication cost reduction.

Graph edge partition has been less studied in literature than graph vertex partition. The Powergraph [3] work is the first work that showed edge partition improves work balancing and reduces communication cost in practice, especially for power-law graph. Bourse, Lelarge, and Vojnovic [1] established the first theoretical approximation guarantee and developed an efficient streaming edge partition algorithm. The target application for both studies is data analytics in large-scale distributed computing clusters, in which case single-pass streaming edge partition algorithms are desired.

The hypergraph partition approach [4] is an indirect approach to solve edge partition problem. The target application for hypergraph partition is parallel program that has potentially good data reuse and has multi-pass computation phases, for instance, sparse linear algebra solvers for optimization problems.

The streaming edge partition algorithms $[1,3]$ are for scenarios when the input graph is given as an stream or only one pass through the input graph is allowed. In these settings their use is much preferred even when they do not yield the same partition quality as the hypergraph algorithm, their non-streaming counterpart. The reason is the large overhead of the latter.

We introduce a novel edge partition model that yields similar or improved partition quality than the hypergraph model, while in the meantime, keeps partition overhead low. Our approach improves previous work in both theory and practice, in the following ways. 

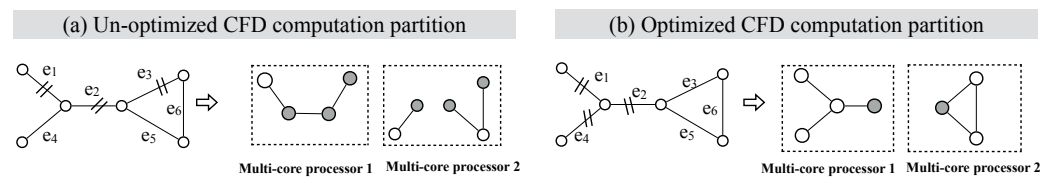

Figure 1: Mapping of $c f d$ interaction computation into two multi-core processors. Assuming we have two multi-core processors and each multi-core processor is in charge of three edges. In (a) there are three fluid elements that need to be copied into both processors while in (b) there is only one.

First, our approach is practical. It has significantly lower partition overhead than the hypergraph partition approach. Partitioning a power-law graph with 2 million vertices and 16 million edges takes about 400 seconds if we apply the best hypergraph algorithm we are aware of, while less than 18 seconds if we apply our model.

Second, our method is simple and easy to implement. It is based on a transformation procedure name split-and-connect procedure we developed in this work. The split-and-connect procedure improves the partition quality (the number of vertex copies in different partitions) over the streaming and the hypergraph approaches or is at least similar to the better of the two. In particular, when compared with the streaming edge partition algorithms, the partition quality improvement is the most pronounced in power-law graphs.

Third, it provides a theoretical guarantee for graphs with all ranges of parameters. Similar to the work by Bourse, Lelarge, and Vojnovic [1], by formulating the edge partition problem into vertex partition problem, we establish an approximation guarantee of $O\left(d_{\max } \sqrt{\log n \log k}\right)$, where $n$ is the number of vertices, $m$ is the number of edges, $k$ is the number of partitions, and $d_{\text {max }}$ is the maximum degree. The work by Bourse, Lelarge and Vojnovic [1] showed that this approximation factor holds for graphs with the constraint $m=\Omega\left(k^{2}\right)$. We rigorously proved this approximation factor holds for all graphs.

\section{USE CASES}

We demonstrate how to use edge partition model to improve data reuse in GPU programs under two scenarios: (1) load-balanced work partition model, and (2) data-balanced work partition model. The former corresponds to the Edge-balanced Edge-Partition (E-EP) Problem Vertex-balanced Edge-Partition (V-EP) Problem For both models, the input is a list of 2-tuple modeled as a work graph and the output is a set of work (edge) partitions. We formally define these two work partition models as follows.

Edge-balanced Edge-Partition (E-EP) Problem Given a graph $G=(V, E)$ with the set of vertices $V$ and the set of edges $E$, and vertex capacity constraint $T$. Let $x=\left\{e_{1}, e_{2}, \ldots e_{k}\right\}$ denote a partition of the edges of $G$ into $k$ disjoint subsets, let $\left|e_{i}\right|$ denote the number of unique edges in $e_{i} . \forall n \in V$ and $|E|$ denote the number of edges in $E . P(n)$ is defined as the number of subsets that n's incident edges fall into. We optimize the total vertex replication cost:

$$
\begin{array}{ll}
\underset{x}{\operatorname{minimize}} & R(x)=\sum_{n \in V}(P(n)-1) \\
\text { subject to } & \forall i \in[1 . . k],\left|e_{i}\right| \leq|E| / k
\end{array}
$$

The Edge-balanced Edge-Partition (E-EP) problem has been used to minimize communication while balancing the load between computation threads or nodes. It has been used in the distributed graph processing setting $[1,3]$. We use it to model workload balancing across thread blocks on GPUs [5].

Vertex-balanced Edge-Partition(V-EP) Problem Given a graph $G=(V, E)$ with the set of vertices $V$ and the set of edges $E$, and vertex capacity constraint $T$. Let $x=\left\{e_{1}, e_{2}, \ldots e_{k}\right\}$ denote a partition of the edges of $G$ into $k$ disjoint subsets, and let $V\left(e_{i}\right)$ denote the set of unique vertices in $e_{i} . \forall n \in V$, let $P(n)$ denote the number of subsets that $n$ 's incident edges fall into. We optimize the total vertex replication cost:

$$
\begin{array}{cl}
\underset{x}{\operatorname{minimize}} & R(x)=\sum_{n \in V}(P(n)-1) \\
\text { subject to } & \forall i \in[1 . . k],\left|V\left(e_{i}\right)\right| \leq T
\end{array}
$$

The V-EP model also minimizes vertex replication cost. While the E-EP model aims to balance the workload among threads/processors in space, the V-EP model aims to keep data fit into cache (the capacity constraint $\mathrm{T}$ ) and maximize data reuse over time. We use the E-EP model to perform software throttling on GPUs [2].

\section{CONCLUSION}

We evaluate the effectiveness of our edge partition model over GPU applications from the domains of linear algebra, graph processing, machine learning and scientific simulation. As far as we know, this is the first systematic study of edge partition model for GPU computing. In both settings: load-balanced work partition and databalanced partition, our approach significantly improves data reuse in cache and greatly enhanced program performance. Evaluated on over 200 real-world large sparse matrices and graphs that suffer from cache contention, our technique achieves up to $6.45 \mathrm{X}$ speedup, and a maximum performance loss $\leq 5 \%$.

\section{REFERENCES}

[1] Florian Bourse, Marc Lelarge, and Milan Vojnovic. 2014. Balanced Graph Edge Partition. In Proceedings of the 20th ACM SIGKDD International Conference on Knowledge Discovery and Data Mining (KDD '14). ACM, New York, NY, USA, 1456-1465. https://doi.org/10.1145/2623330.2623660

[2] Yanhao Chen, Ari B. Hayes, Chi Zhang, Timothy Salmon, and Eddy Z. Zhang. 2018. Locality-Aware Software Throttling for Sparse Matrix Operation on GPUs. In 2018 USENIX Annual Technical Conference (USENIX ATC 18). USENIX Association, Boston, MA.

[3] Joseph E Gonzalez, Yucheng Low, Haijie Gu, Danny Bickson, and Carlos Guestrin. 2012. PowerGraph: Distributed Graph-Parallel Computation on Natural Graphs.. In OSDI, Vol. 12. 2.

[4] Bruce Hendrickson and Tamara G. Kolda. 2000. Graph Partitioning Models for Parallel Computing. Parallel Comput. 26, 12 (Nov. 2000), 1519-1534. https: //doi.org/10.1016/S0167-8191(00)00048-X

[5] Lingda Li, Robel Geda, Ari B. Hayes, Yanhao Chen, Pranav Chaudhari, Eddy Z. Zhang, and Mario Szegedy. 2017. A Simple Yet Effective Balanced Edge Partition Model for Parallel Computing. Proc. ACM Meas. Anal. Comput. Syst. 1, 1, Article 14 (June 2017), 21 pages. https://doi.org/10.1145/3084451 